\title{
Diagnosis and Treatment of Inflammatory Root Resorption by Intentional Reimplantation
}

\author{
Suresh Kumar Saini, Poonam Bogra, Amita Saini
}

\begin{abstract}
External root resorption is a lytic process occurring in the cementum or cementum and dentin of the roots of teeth. It is frequently caused by trauma. Such cases are often difficult to diagnose especially if lesion is on buccal or lingual side, and at times may be difficult to treat using conventional root canal treatment, with extraction being left the only alternative. This article describes the diagnosis and treatment of a case of inflammatory resorption of palatal wall of palatal root of maxillary left first molar by intentional reimplantation. The provisional diagnosis was made from clinical and radiographic observations. The treatment consisted of intentional reimplantation. After extraction it was found that palatal wall of palatal root had been resorbed from furcation till apex. After the resection of the palatal root at the point of resorption, tooth was obturated, reimplanted and splinted. This tooth is serving well since 5 years.
\end{abstract}

Keywords: Reimplantation, Rootresorption, Correct diagnosis.

How to cite this article: Saini SK, Bogra P, Saini A. Diagnosis and Treatment of Inflammatory R oot Resorption by Intentional Reimplantation. Int J Experiment Dent S ci 2012;1(2):106-109.

Source of support $\mathrm{Nil}$

Conflict of interest: None declared

\section{INTRODUCTION}

Inflammatory resorption is one of the complications of traumatized teeth which may occur as a result of damage to the surrounding protective tissues. ${ }^{1} \mathrm{~A}$ lthough, radiographic examination is an important adjunct in clinical diagnosis of external root resorption, its efficacy is limited by various factors including size and location of the defect. Most of the defects located on palatal and buccal side often go undetected and may lead to perforation which drastically reduces the possibility of conventional surgical and nonsurgical treatment, with extraction being the only treatment.

It is a well known fact that dental reimplantation is indicated following traumatic avulsion by the preservation of cellular vitality in the periodontal ligament and under conditions of asepsis. Indications for intentional reimplantation are as follows:

- In areas of difficult access like lower second molars because of bone thickness or any perforation on palatal root of maxillary bicuspid as a large portion of buccal bone and root must be removed to gain access to palatal root. $^{2}$

- In areas where vital anatomical structures makes the traditional procedure difficult. ${ }^{2}$
- In patients who are unable to keep their mouth open for the necessary length of time. ${ }^{3}$

- When perforating internal or external resorption is present, yet surgery is impractical. $^{3}$

- Typical complications, after severe traumatic injuries are localized areas of inflammatory root resorption. These can be considered as areas of periodontal pathology below the attachment level. Treatment of these defects is extremely difficult without affecting the periodontal health of adjacent teeth. Intentional reimplantation has been suggested in these cases because these difficult to access areas can be maximally treated while the tooth is out of mouth without damaging the attachment in adjacent teeth. ${ }^{4}$

The success rate of intentional reimplantation in posterior teeth varies greatly. However, intentional dental reimplantation is an effective strategy for the treatment of teeth that would be difficult, if not impossible, to treat using conventional endodontic or surgical treatment. This report describes a clinical case where intentional reimplantation was chosen as a treatment to manage a case of palatal root resorption in maxillary first molar.

\section{CASE REPORT}

A 30-year-old male patient reported in the Department of Conservative D entistry with the chief complaint of pain on chewing since last 1 to 1.5 months in one of the posterior teeth of left side of upper jaw. There was history of trauma that took place 5 months back due to which bilateral condylar fracture occurred that had been operated. Patient was having pain on percussion in 26 , on palpation palatal mucosa was painful; and tooth was having second degree of mobility. The tooth gave no response when tested by electric pulp tester. Radiographic examination revealed periapical radiolucency around mesiobuccal and distobuccal roots. On radiograph only a faint outline of pal atal root could be made out and the radiopacity of the root was less as compared to mesiobuccal and distobuccal roots (Fig. 1).

Patient was advised to undergo root canal treatment of 26. A ccess opening was made and the canals were located. In palatal canal even 60 number file was loose and there was bleeding from canal on insertion of instrument. It was suspected that file was going either in the perforation or palatal root had resorbed. A diagnostic radiograph (Fig. 2) 


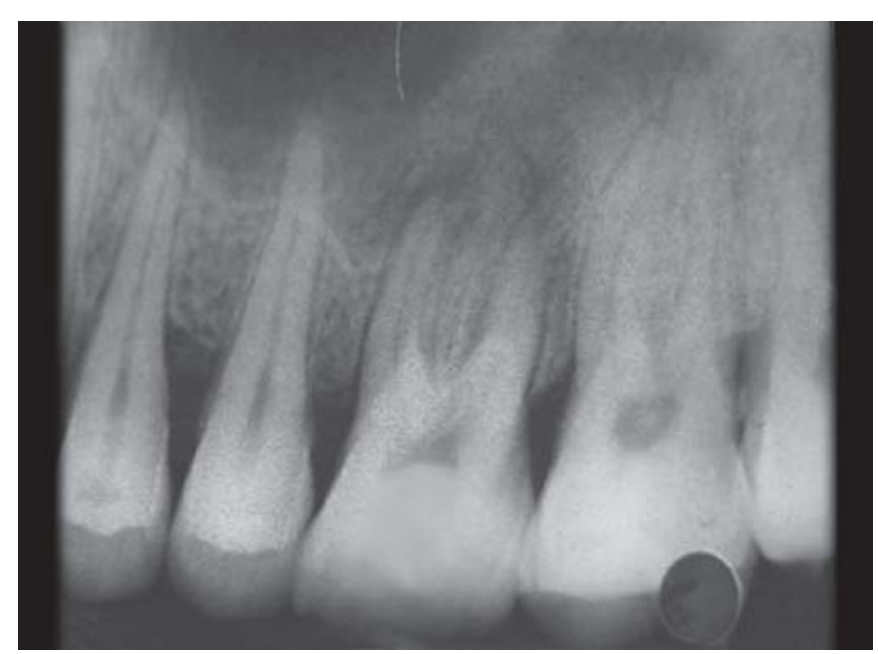

Fig. 1: Preoperative radiograph showing faint outline of palatal root

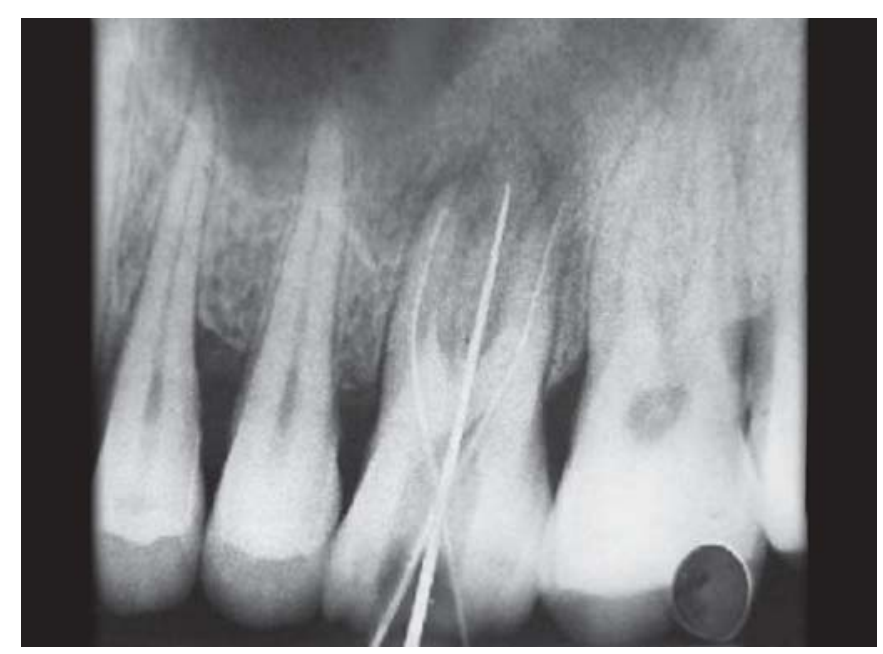

Fig. 2: Diagnostic intraoral periapical X-ray

was taken with files in mesiobuccal and distobuccal canals and 80 number gutta-percha cone in palatal canal. On the basis of radiograph and clinical judgment it was confirmed that gutta percha was located in palatal canal.

As there was no evidence of apical stop on tactile examination in palatal canal, working length $(20 \mathrm{~mm})$ was established on basis of radiograph and working length of buccal canals which were $19 \mathrm{~mm}$ each. Palatal canal was bleeding during instrumentation. Canals were heavily irrigated with saline and chlorhexidine. $\mathrm{NaOCI}$ was not used for irrigation as it was suspected that palatal root had at least partially resorbed. Chlorhexidine and calcium hydroxide dressing was given for 3 weeks which was replaced after every week. A fter that, patient's symptoms subsided and there was no bleeding from palatal canal. At this stage tooth was obturated with gutta percha and zinc oxide eugenol sealer (Fig. 3).

On the next day of treatment patient reported back to the department with the complaint of pain and swelling. At

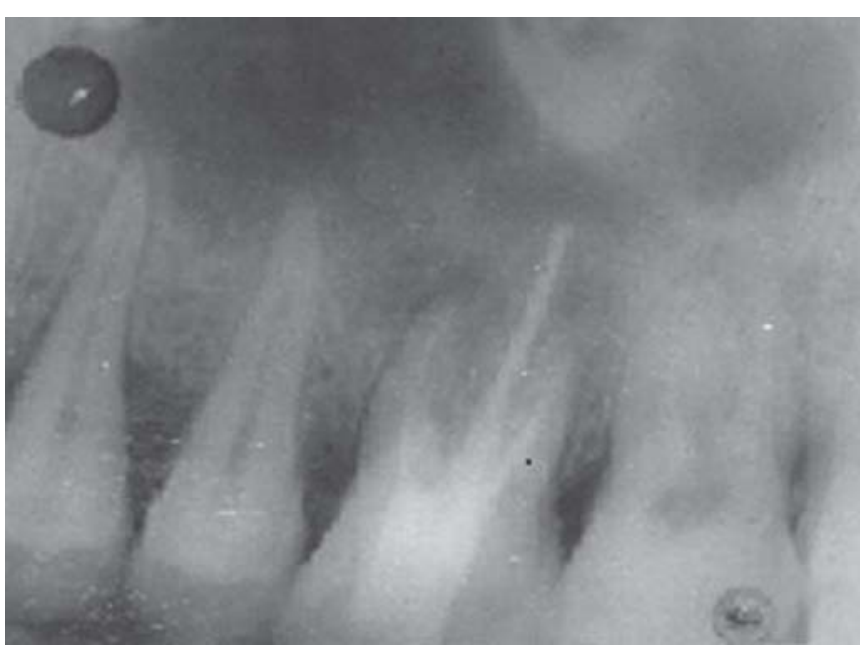

Fig. 3: Radiograph after initial obturation

that time root canal filling was removed and profuse bleeding was observed from palatal canal. It was suspected that palatal root was resorbed and considering all other factors, two treatment options were left, either intentional reimplantation or extraction followed by prosthesis. Patient opted for intentional reimplantation.

\section{PROCEDURE}

One hour before procedure, patient was given antibiotic coverage and premedication. The patient was prepared for surgery and local anesthesia was administered. The tooth was extracted with minimal trauma by use of forceps (Fig. 4). Examination of the extracted tooth revealed that palatal root was shorter than mesiobuccal and distobuccal roots and the palatal wall of palatal root was resorbed from furcation area till apex (5 mm) (Fig. 5), whereas buccal wall was intact throughout the root (approximately $16 \mathrm{~mm}$ in length). Palatal root was resected at the point of resorption (Fig. 6) and the remaining roots were obturated with gutta percha and zinc oxide sealer.

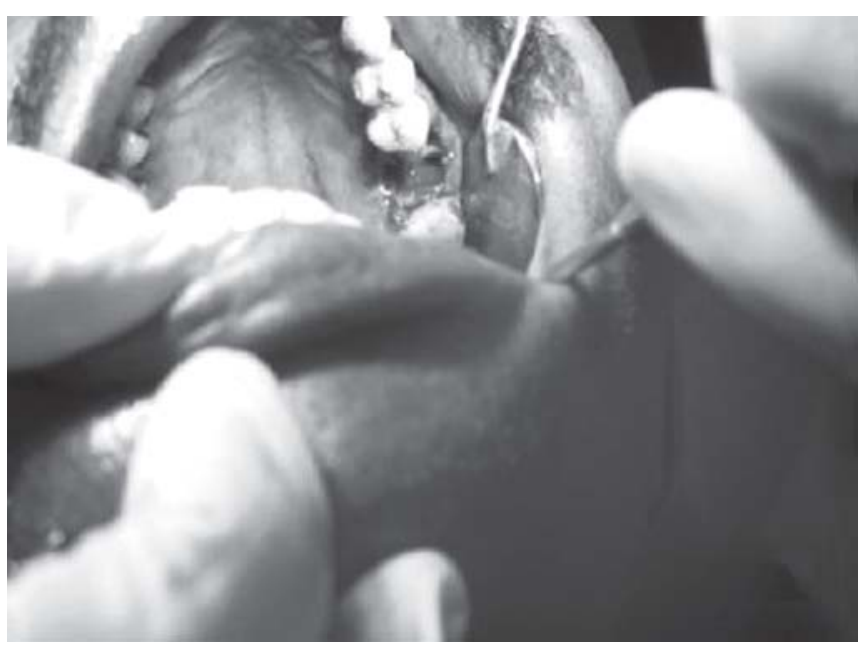

Fig. 4: Socket after extraction with minimal trauma 


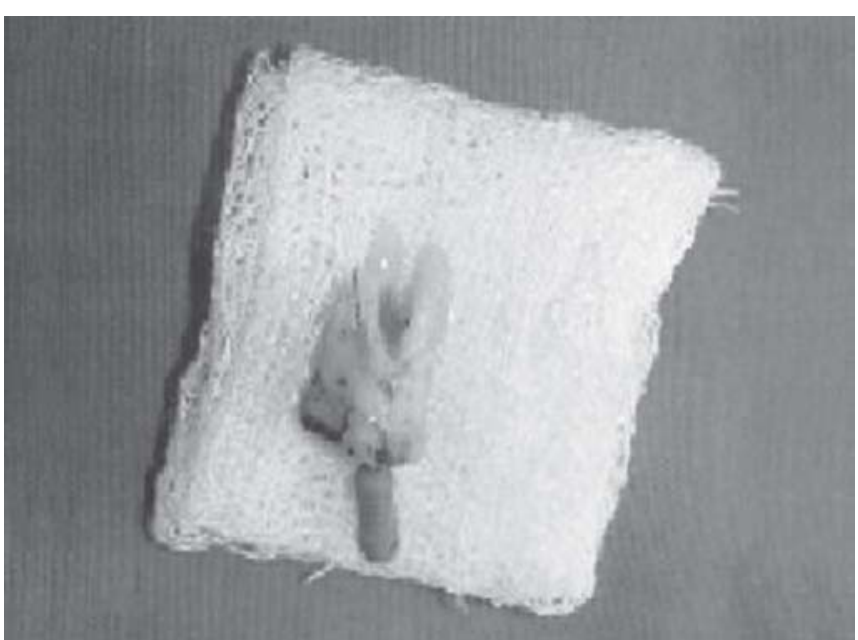

Fig. 5: Instrument indicating resorbed palatal root

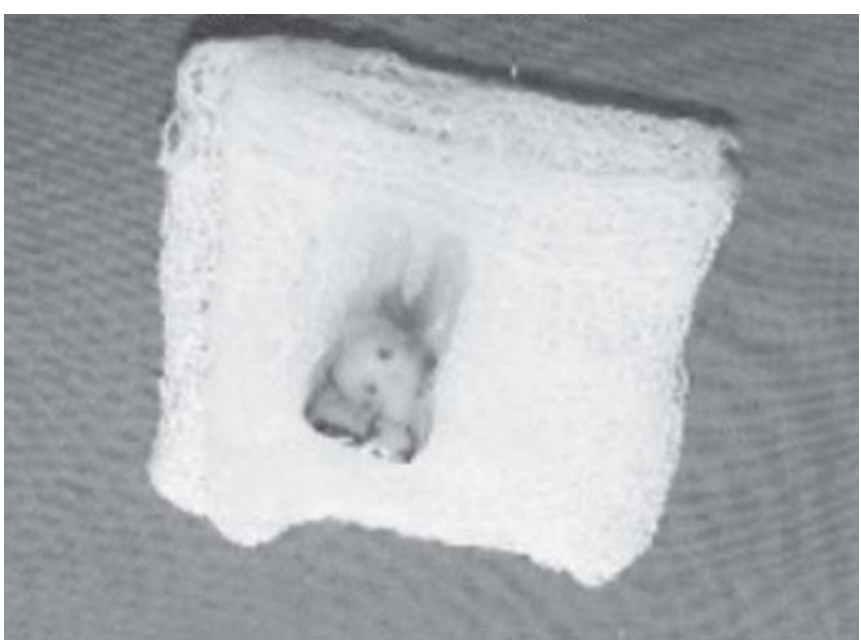

Fig. 6: Resected palatal root at the point of resorption

During the procedure care was taken not to damage the periodontal ligament. A fter obturation tooth was replanted in to the socket in less than 30 minutes from extraction. Essig's wiring was done to stabilize the tooth (Fig. 7). The occlusion was not reduced during and after the surgery in an effort to promote healing. Postoperative instructions were given to the patient. On next day, tooth was permanently restored with silver amalgam.

Patient was asked to report 15 days but he turned up 37 days later, at that time wiring was removed but tooth was still mobile. It was again splinted for 15 days. Splint was removed after 15 days, when mobility of the tooth had greatly reduced. After 5.5 months of recall radiograph (Fig. 8), it showed good healing. Patient is asymptomatic after 5 years, although radiograph showed root resorption (Fig. 9).

\section{DISCUSSION}

Root resorption occurs when there is loss or alteration of the protective layer (predentine or precementum) along with

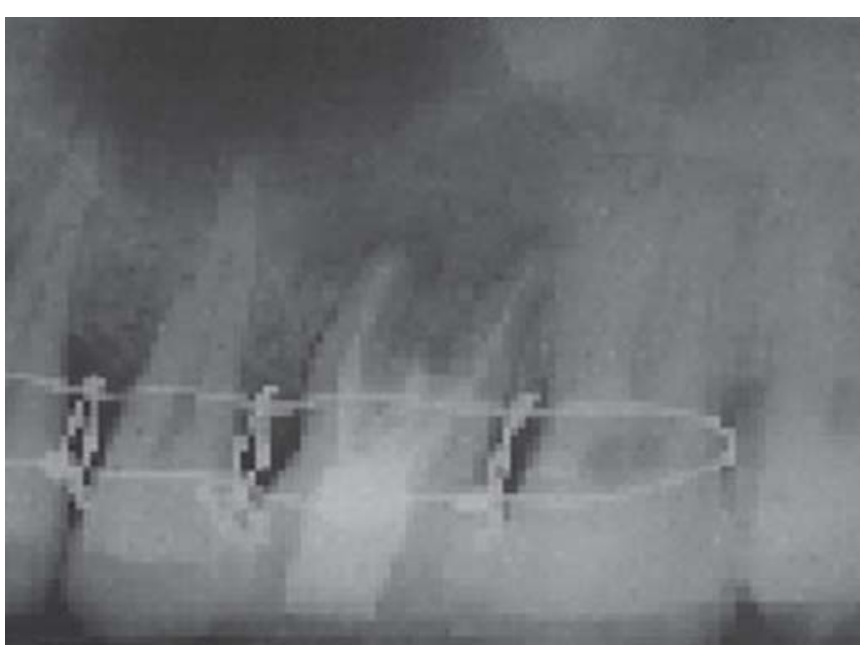

Fig. 7: Splinted tooth in place

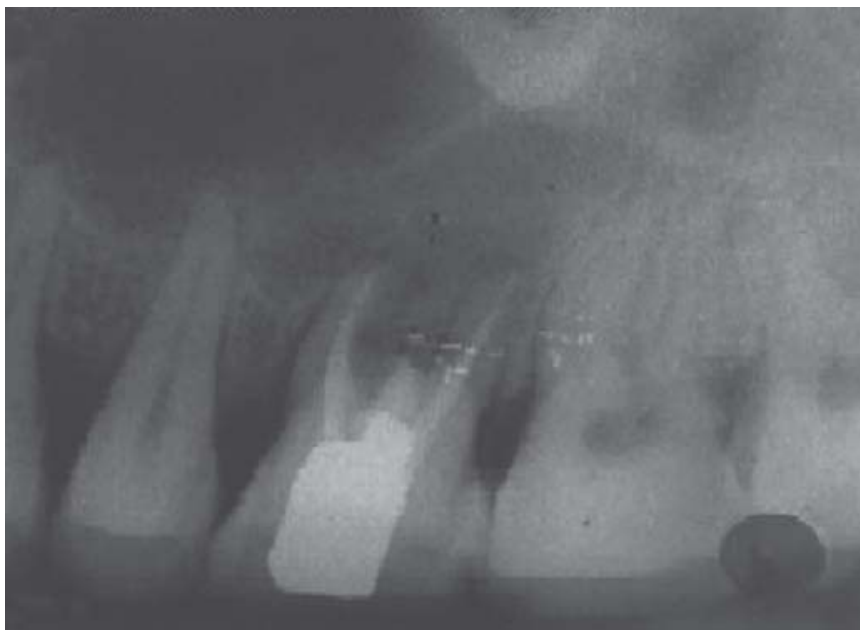

Fig. 8: Follow-up radiograph after 5.5 months

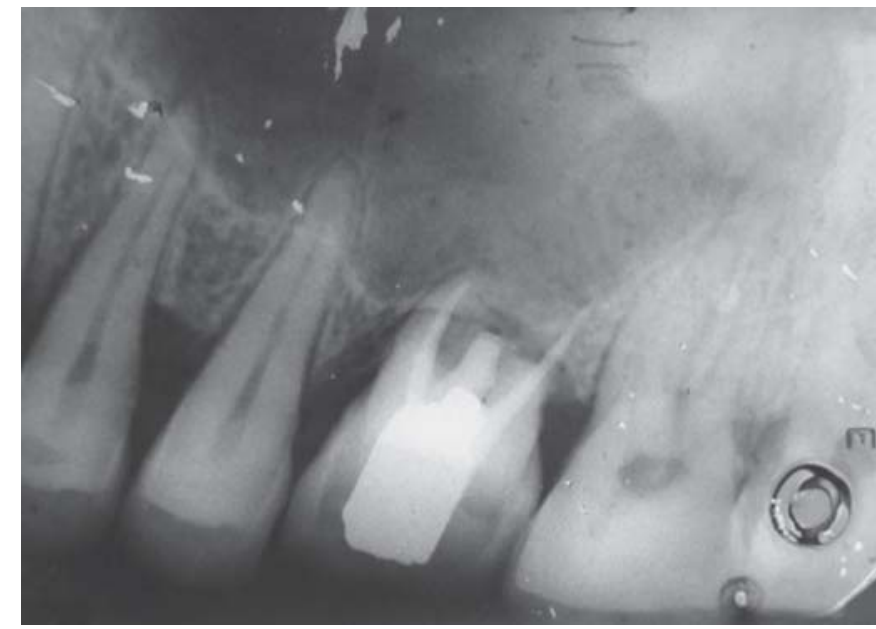

Fig. 9: Follow-up radiograph after 5 years

inflammation of the unprotected root surface. Damage to protective layer can occur either directly due to trauma or indirectly, when an inflammatory response occurs as a result of dental injury. ${ }^{1}$ Incidence of root resorption as a result of traumatic injury ranges from 4 to $86 \% .^{5}$ 
Diagnosis and Treatment of Inflammatory Root Resorption by Intentional Reimplantation

In such cases treatment principles include the prevention or elimination of the stimulus for the periradicular inflammation, i.e. prevention and elimination of pulp space bacteria. ${ }^{1}$ In the present case, most probable reason for resorption of palatal root was trauma causing inflammation, and patient reported 5 to 6 months after trauma, at time when root resorption had al ready established. To diagnose such cases with radiograph is a dilemma especially when one wall is resorbed and other is intact and to determine the extent of resorption is difficult if not impossible, therefore, such teeth are extracted. Tuned aperture computer radiography is a useful tool for the diagnosis of facial or lingual/palatal defects which was most often missed during radiographic examination. ${ }^{6}$ In the present case, definitive diagnosis of resorption of palatal wall of palatal root could not be done because of unavailability of equipment.

Although provisional diagnosis was made from clinical and radiographic observations, while making treatment plan it was not diagnosed exactly up to which point root was resorbed. As nonsurgical root canal treatment was not successful and surgery was not possible because of three reasons. First, patient was not able to keep his mouth open for long as he has undergone condylar surgery recently. Second, surgery from palatal side was difficult to perform because of proximity to greater palatine artery. Third, in gaining access to palatal root through buccal approach healthy buccal bone had to be sacrificed. Therefore, intentional reimplantation was considered as the treatment of choice and tooth was retained for a considerable period of time. At 5.5 months of follow-up, no pain or mobility was observed. Radiograph showed no sign of resorption. A fter 5 years tooth appeared to function normally. Patient was asymptomatic and tooth showed grade I mobility, although radiograph showed replacement root resorption. Extraction should be considered as the last resort as following tooth extraction, $25 \%$ decrease in bone volume was seen during the first year and 40 to $60 \%$ loss in width occurs within 3 years after tooth loss. ${ }^{7,8}$ A fter intentional reimplantation even if replacement resorption occurs, there will be a bony fill in the area and the height of alveolar bone will be partially restored. This bony fill may also make the future prosthesis or implant easier and more esthetic. ${ }^{4}$

The success of intentional reimplantation is likely dependent upon a minimally traumatic extraction, short extraoral time with postoperative patient compliance. ${ }^{9}$ Every effort should be made to keep periodontal ligament viable by moistening the tooth frequently in sterile solution, anesthetic solution or milk. ${ }^{10}$

\section{CONCLUSION}

Preservation of tooth in the mouth for as long as possible is the major goal in any conservative treatment. Dental reimplantation is certainly not the primary therapy of choice in case of endodontic failure, not all seemingly hopeless cases are amenable to intentional reimplantation. At the same time, a respectable degree of success can be attained, if prudent endodontic surgeon will carefully consider the preoperative factors, minimize the midtreatment factors, and attempt to control the postoperative factors in order to enhance the success of this last resort approach for tooth maintenance.

\section{REFERENCES}

1. Trope M. Root resorption due to dental trauma. Endodontic Topics 2002;1:79-100.

2. W eine FS. Endodontic therapy. St L ouis: CV M osby 2004:229.

3. K ratchman S. Intentional reimplantation. Dent $\mathrm{Clin} \mathrm{N}$ orth $\mathrm{A} m$ 1997;41(3):603-17.

4. Demiralp B, N ohutçu RM, Tepe DI, Eratalay K. Intentional replantation for periodontally involved hopeless teeth. Dent Traumatol 2003;19:45-51.

5. Bender IB, Rossman LE. Intentional replantation of endodontically treated teeth. Oral Surg Oral M ed Oral Pathol 1993 N ov;76(5):623-30.

6. N ance RS, Tyndall D, L evin L G, Trope M. Diagnosis of external root resorption using TACT (tuned-aperture computed tomography). Endod Dent Traumatol 2000;16(1):24-28.

7. Carlsson GE, Thilander H, Hedegard B. Histological changes in the upper alveolar process after extractions with or without insertion of an immediate full denture. Acta Odontol Scand 1967;25:21-43.

8. Piertokovski J, M assler M. A lveolar root resorption following tooth extraction. J Prosthet D ent 1967;17:21-27.

9. Cotter M R, Panzarino J. Intentional replantation: A case report. J Endod 2006;32(6):579-82.

10. Grossman LI. Endodontic practice (11th ed). India, Vargnese House 1991:34-38.

\section{ABOUT THE AUTHORS}

\section{Suresh Kumar Saini (Corresponding Author)}

Reader, Department of Prosthodontics, BRS Dental College Panchkula, Haryana, India, e-mail: drsksaini@yahoo.com

\section{Poonam Bogra}

Professor, D epartment of Endodontics, DA V Dental College, Y amuna Nagar, Haryana, India

\section{Amita Saini}

Reader, D epartment of Endodontics, BRS D ental College, Panchkula Haryana, India 